\title{
Estudio de un Sistema de Tratamiento de Aguas Residuales Provenientes de una Industria de Papel
}

\author{
Merizalde, Edgar'; Montenegro, Lucía'; Cabrera, Marcelo \\ ${ }^{1}$ Escuela Politécnica Nacional, Facultad de Ingeniería Química y Agroindustria, Quito, Ecuador
}

\begin{abstract}
Resumen: El objetivo de la presente investigación es estudiar un sistema de tratamiento de aguas residuales provenientes de una industria de papel con base en un reactor biológico rotativo de contacto (RBC). Se caracterizó el agua residual llegando a la conclusión que dicho efluente no se puede descargar directamente debido a que incumple en las concentraciones establecidas en el Registro Oficial N ${ }^{\circ} 387$ del 4 de Noviembre del 2015 de la República del Ecuador en los parámetros de DBO, DQO, sólidos sedimentables, suspendidos y totales. Se inició el estudio con un tratamiento de sedimentación a escala de laboratorio sin la adición de floculantes y coagulantes lo que permitió la remoción de sólidos sedimentables. Luego pasó a un RBC cuyo volumen inicial fue de 3,0 L: 2,7 L de agua residual y 0,3 L de inóculo de microorganismos; el tiempo de estabilización de los microorganismos fue de 14 días. Posteriormente se tuvo un tratamiento por medio de un filtro abierto de arena y grava a escala de laboratorio con una altura de $55 \mathrm{~cm}$ de arena de sílice precipitada. Estos tratamientos permitieron el cumplimiento de la normativa ambiental para el control de descargas líquidas. Se diseñó el sistema de tratamiento para un flujo de $140 \mathrm{~m}^{3} / \mathrm{dí}^{3}$, el cual cuenta con un tanque de almacenamiento de $32,67 \mathrm{~m}^{3}$, un sedimentador de $30,00 \mathrm{~m}^{3}$, un RBC de $35,95 \mathrm{~m}^{3}$ con 150 discos de polietileno y dos filtros abiertos de arena y grava de $11,66 \mathrm{~m}^{3}$.
\end{abstract}

Palabras clave: reactor biológico rotativo de contacto (RBC), contaminantes al agua, inóculo.

\section{Study of a Wastewater Treatment System from a Paper Industry}

\begin{abstract}
This project aims to study a wastewater treatment system from a paper industry based on a rotating biological contact reactor (RBC). The wastewater was characterized, concluding that the effluent can't be discharged directly because it does not comply with the concentrations established in Registro Oficial $\mathrm{N}^{\circ} 387$ of November 4 , 2015 of the Republic of Ecuador in the parameters of BOD, COD, settle able, suspended and total solids. The study started with a sedimentation treatment on a laboratory scale without the addition of flocculants and coagulants, which allowed the removal of settle able solids. Then it went to a RBC whose initial volume was 3.0 L: 2.7 L of wastewater and $0.3 \mathrm{~L}$ of inoculum of microorganisms; the stabilization time of the microorganisms was 14 days. Subsequently, a treatment was carried out by means of an open filter of sand and gravel on a laboratory scale with a height of $55 \mathrm{~cm}$ of precipitated silica sand. These treatments allowed compliance with environmental regulations for the control of liquid discharges. The treatment system was designed for a flow of $140 \mathrm{~m}^{3} / \mathrm{day}$, which has a storage tank of 32.67 $\mathrm{m}^{3}$, a settler of $30.00 \mathrm{~m}^{3}$, a RBC of $35.95 \mathrm{~m}^{3}$ with 150 polyethylene disks and two open sand and gravel filters of $11.66 \mathrm{~m}^{3}$.
\end{abstract}

Keywords: rotating biological contactor reactor (RBC), water contaminants, and inoculum.

\section{INTRODUCCIÓN}

La industria de papel tiene un alto consumo de agua en sus procesos de producción, generando lodos que están formados principalmente por pulpa de celulosa, un polímero que proviene de la madera (Casey, 2000).

El agua arrastra y concentra contaminantes, compuestos químicos y materia orgánica producida en los diferentes procesos; por esta razón, si el efluente no es tratado de una forma adecuada puede aportar un alto impacto ambiental al recurso agua (Castillo et al., 2012).
La industria de producción de papel tisú tiene un consumo de agua de $40 \mathrm{~m}^{3} / \mathrm{t}$, una generación de sólidos suspendidos de 7 $\mathrm{kg} / \mathrm{t}$ y una DQO de $55 \mathrm{~kg} / \mathrm{t}$, aproximadamente (Casey, 2000).

El Registro Oficial No 387 del 4 de Noviembre del 2015 de la República del Ecuador obliga a mantener un control de los residuos que se producen por las actividades industriales y que implique grandes impactos al ambiente o a la calidad de vida de los habitantes, garantizando el buen vivir de todos los ciudadanos.

El reactor biológico rotativo de contacto (RBC) ha sido durante muchos años uno de los tratamientos más eficientes

merizaldeomar66@gmail.com

Recibido: 09/02/2018

Aceptado: 03/01/2019

Publicado: 30/04/2019 
para depurar aguas residuales con alto contenido de carga orgánica. La remoción de DBO y DQO varía entre 80 y $95 \%$ (Behling et al., 2008), por tal motivo se ha usado como parte del tratamiento del agua proveniente de una industria de papel.

El RBC está formado por discos inertes de polietileno, que se encuentran divididos por etapas a lo largo de un eje longitudinal que giran entre 1 y 4 RPM con la ayuda de un motor rotativo. En la superficie de los discos se empieza a formar una biopelícula de microorganismos que son los responsables de la depuración del agua residual (Deloya, 2001).

El presente artículo expone los resultados obtenidos al realizar un estudio de un sistema de tratamiento del agua residual con base en un sedimentador, un reactor biológico rotativo de contacto y un filtro abierto de arena y grava para dar una solución efectiva al tratamiento y poder cumplir con las normas técnicas para el control de descargas líquidas que se exige en el Ecuador.

\section{MATERIALES Y MÉTODOS}

\subsection{Materiales}

Todos los reactivos químicos empleados fueron de calidad analítica. El agua fue destilada antes de su utilización. El agua residual tratada fue de una fábrica productora de papel tisú.

Las pruebas de sedimentación a escala de laboratorio se realizaron con ayuda de conos Imhoff de $1 \mathrm{~L}$ y una probeta de $1 \mathrm{~L}$.

En el estudio del RBC se construyó un equipo a escala de laboratorio mediante discos de polietileno, un motor rotativo y un tanque de PVC como se observa en la Figura 1.

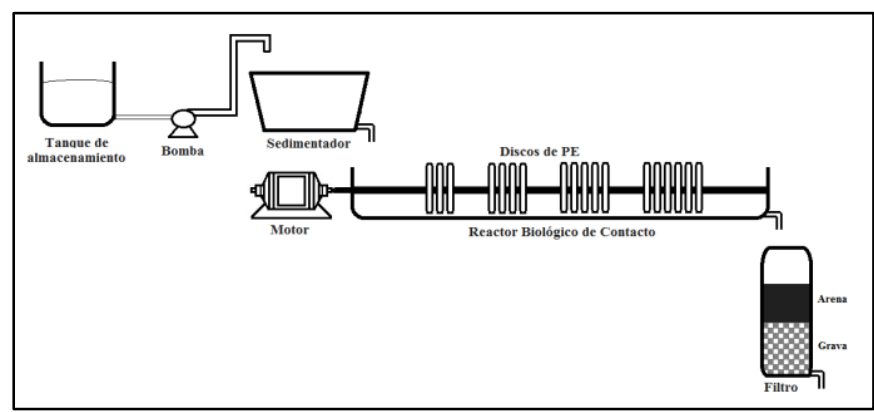

Figura 1. Esquema del tratamiento a escala de laboratorio

El inóculo para el RBC se obtuvo del licor del proceso de una industria maderera y los nutrientes para los microorganismos a partir de nitrato de potasio al $10 \%$.

Para las pruebas de filtración a escala de laboratorio se construyó un filtro de arena y grava mediante una tubería PVC de 6 in de diámetro, arena de sílice precipitada y grava.

\subsection{Métodos}

Las mediciones experimentales de los ensayos para los tratamientos se realizaron por triplicado. Con los resultados obtenidos se determinaron los valores promedio y las desviaciones estándar. Se aceptaron los resultados cuando las desviaciones estándar fueron menores al $10 \%$.

2.2.1 Caracterización físico-química de las muestras de aguas residuales

Se realizaron análisis de los parámetros físico-químicos del agua residual mediante los Standard Methods for the Examination of Water and Wastewater (APHA, 2012) para DBO, DQO y sólidos: suspendidos, totales y sedimentables. Los análisis se realizaron en el Laboratorio Nacional de Aguas y Sedimentos.

2.2.2 Evaluación del tratamiento de sedimentación a escala de laboratorio

a. Determinación de la altura de interfaz del líquido clarificado en la sedimentación

Se midió en una probeta $1 \mathrm{~L}$ de agua residual, luego se trasvasó a un cono Imhoff de $1 \mathrm{~L}$; se registraron datos de concentración de sólidos sedimentables para diferentes tiempos: 5, 15, 25, 40, 55, 70, 90 y 120 min y se reportó en $\mathrm{mL} / \mathrm{L}$. (Davis, 2010)

b. Determinación de los sólidos suspendidos en la sedimentación

Se procedió a tomar $9 \mathrm{~L}$ de agua residual y se distribuyó en 9 vasos de precipitación de $1 \mathrm{~L}$ de muestra cada uno. Se procedió a tomar una muestra de $100 \mathrm{~mL}$ del sobrenadante con la ayuda de una pipeta volumétrica para cada vaso de precipitación a tiempos de: 0, 5, 15, 25, 40, 55, 70, 90 y 120 min. Se realizó el análisis de sólidos suspendidos mediante el procedimiento APHA 2540 D.

\section{c. Determinación del tiempo de sedimentación}

Para determinar el tiempo de sedimentación a escala de laboratorio se realizó con base en la norma APHA 2540 F. Se colocó el agua residual en un cono Imhoff de 1 L, se registraron datos de concentración de sólidos sedimentables para diferentes tiempos: 5, 15, 25, 40, 55, 70, 90 y 120 min y se reportó en $\mathrm{mL} / \mathrm{L}$, con tres repeticiones para cada tiempo.

2.2.3 Evaluación del reactor biológico rotativo de contacto (RBC) a escala de laboratorio

a. Conteo y estabilización de los microorganismos en el RBC

El inóculo para el RBC se obtuvo del licor del proceso de una industria maderera y se realizó un conteo de microorganismos mediante cámaras de recuento de Petroff Hauser (GAB System, 2005).

La muestra inicial se preparó para $3 \mathrm{~L}$ de mezcla, compuesta por agua residual en un $90 \%(\mathrm{v} / \mathrm{v})$ y el inóculo de microorganismos $10 \%(\mathrm{v} / \mathrm{v})$. Estas concentraciones según Romero (2016) son ideales para iniciar un proceso de estabilización entre el agua residual y un inóculo de microorganismos. 
Para estabilizar el RBC, se encendió el reactor con la muestra inicial preparada y se dosificó $0,7 \mathrm{~mL} / \mathrm{min}$ de agua residual, proveniente del tratamiento de sedimentación, el reactor funcionó de forma continua. El caudal permitió un contacto íntimo entre los discos y el agua residual, se tomó una muestra de 1 L cada día y se procedió a caracterizar las variables de salida como DBO y DQO. El RBC a nivel de laboratorio tuvo una capacidad de $5 \mathrm{~L}$.

\section{b. Determinación del flujo inicial de agua residual al RBC}

Una vez estabilizados los microorganismos se realizaron ensayos a escala de laboratorio a diferentes concentraciones de cargas orgánicas a la entrada del RBC de 35, 45, 55 y 60 g $\mathrm{DQO} / \mathrm{m}^{2 *}$ día, según lo planteado por Romero, 2016. (p. 620). Para cada ensayo se determinaron las concentraciones finales de DQO.

2.2.4 Evaluación de un filtro de arena y grava a escala de laboratorio

a. Caracterización granulométrica de las arenas para el lecho filtrante

Se realizaron pruebas de granulometría para dos tipos de arena sílice: precipitada y fume. Las muestras de arena se vertieron en juego de tamices con un rango de tamaño de poro entre 0,15 $\mathrm{mm}$ a $2,38 \mathrm{~mm}$; y se procedió a tamizar con la ayuda un agitador mecánico.

Se evaluó la arena con mejor distribución granular para un rango de coeficiente de uniformidad entre 0,0 a 2,5; mientras menor sea el coeficiente de uniformidad mejor distribución tendrá la arena sílice (Coulson y Richardson, 2003).

b. Determinación de la altura efectiva del filtro de arena y grava

Se dosificó agua residual proveniente del RBC al filtro para cargas hidráulicas entre $1,74 \times 10^{-5}$ y $2,89 \times 10^{-5} \mathrm{~m} / \mathrm{s}$. Al final de cada ensayo se determinaron los sólidos suspendidos. La altura efectiva del lecho filtrante fue la que mejor remoción de sólidos suspendidos dio como resultado al final del tratamiento (Coulson y Richardson, 2003).

c. Determinación del tiempo de saturación del filtro de arena y grava

Se construyó un filtro de arena y grava a escala de laboratorio mediante una tubería PVC de 6 in de diámetro, la arena de sílice con mejor distribución granular y grava. (Romero, 2016)

Se procedió a dosificar a los filtros diferentes flujos de agua residual tratadas por el $\mathrm{RBC}$, con cargas hidráulicas entre 1,74 x $10^{-5}$ y $2,89 \times 10^{-5} \mathrm{~m} / \mathrm{s}$.

2.2.5 Diseño de un sistema de tratamiento de aguas residuales

Con los resultados obtenidos de las Secciones 2.2.1, 2.2.2, 2.2.3 y 2.2.4; se procedió al diseño de un sistema de tratamiento de aguas residuales (Metcalf y Eddy, 2003).

\section{RESULTADOS Y DISCUSIÓN}

\subsection{Resultados de la caracterización físico-química de las aguas residuales}

Los parámetros que incumplen con los límites permisibles establecidos en el Registro Oficial $N^{\circ} 387$ del 4 de noviembre del 2015 de la República del Ecuador para el control de descargas líquidas hacia un cuerpo de agua dulce, un cuerpo de agua marina o el alcantarillado público son los mostrados en la Tabla 1 (Correa y Tapia, 2015):

Tabla 1. Caracterización de los parámetros del agua residual junto con valores norma.

\begin{tabular}{ccrrrr}
\multicolumn{6}{c}{ Valores norma. } \\
\hline Parámetro & Unidad & $\begin{array}{c}\text { Valor } \\
\text { inicial }\end{array}$ & $\begin{array}{c}\text { Valor } \\
\text { Norma 1 }\end{array}$ & $\begin{array}{c}\text { Valor } \\
\text { Norma 2 }\end{array}$ & $\begin{array}{c}\text { Valor } \\
\text { Norma 3 }\end{array}$ \\
\hline $\mathrm{DBO}$ & $\mathrm{mg} / \mathrm{L}$ & 980,2 & 250 & 100 & 200 \\
$\mathrm{DQO}$ & $\mathrm{mg} / \mathrm{L}$ & 1707,0 & 500 & 200 & 400 \\
$\begin{array}{c}\text { Sólidos totales } \\
\text { Sólidos }\end{array}$ & $\mathrm{mg} / \mathrm{L}$ & 6350,0 & 1600 & 1600 & No espec. \\
$\begin{array}{c}\text { sedimentables } \\
\text { Sólidos }\end{array}$ & $\mathrm{mL} / \mathrm{L}$ & 221,0 & $<1.0$ & $<1.0$ & No espec. \\
suspendidos & $\mathrm{mg} / \mathrm{L}$ & 4591,0 & 220 & 130 & 250 \\
\hline
\end{tabular}

Valor Norma 1: Límites de descarga al sistema de alcantarillado público

Valor Norma 2: Límites de descarga a un cuerpo de agua dulce

Valor Norma 3: Límites de descarga a un cuerpo de agua marina

\subsection{Evaluación del tratamiento de sedimentación a escala de laboratorio}

3.2.1 Determinación de la altura de interfaz del líquido clarificado en la sedimentación

Los sólidos sedimentables entran en un estado de reposo con respecto al agua residual al minuto 90, debido a que la solución está saturada de sólidos con una altura de $0,32 \mathrm{~m}$ y una zona de espesamiento de lodos de $0,18 \mathrm{~m}$, como se observa en la Figura 2.

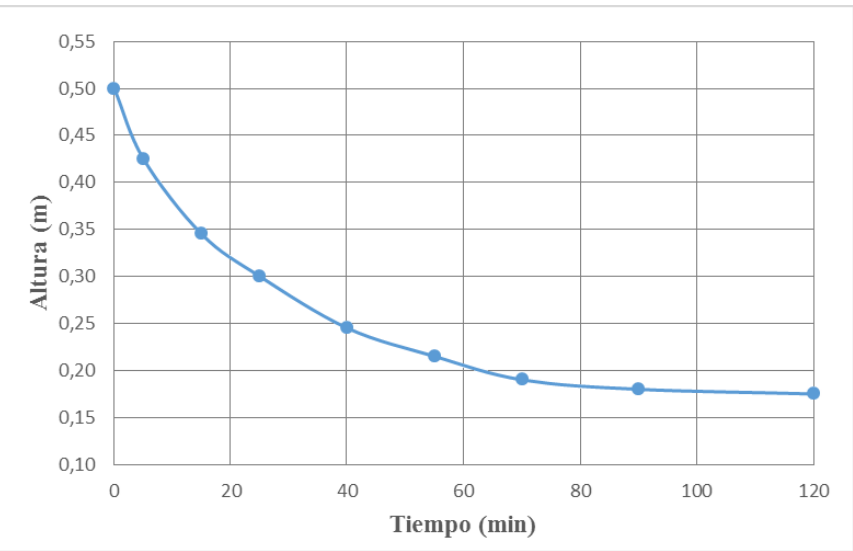

Figura 2. Curva de altura del líquido clarificado en función del tiempo

3.2.2 Determinación de los sólidos suspendidos en la sedimentación

La remoción de sólidos suspendidos se observa en la Tabla 2. 
Tabla 2. Porcentaje de remoción de sólidos suspendidos en el sobrenadante a diferentes tiempos de sedimentación

\begin{tabular}{ccc}
\hline $\begin{array}{c}\text { Tiempo } \\
(\text { min) }\end{array}$ & $\begin{array}{c}\text { Concentración de sólidos } \\
\text { suspendidos }(\mathbf{m g} / \mathbf{L})\end{array}$ & $\begin{array}{c}\text { Remoción de sólidos } \\
\text { suspendidos }(\%)\end{array}$ \\
\hline 0 & 4575 & 0,00 \\
5 & 3256 & 28,83 \\
15 & 2488 & 45,62 \\
25 & 861 & 81,18 \\
40 & 581 & 87,30 \\
55 & 436 & 90,46 \\
70 & 327 & 92,85 \\
90 & 298 & 93,49 \\
120 & 298 & 93,49 \\
\hline
\end{tabular}

La remoción de sólidos fue del 93,49 \%; un valor alto comparado con la concentración inicial, debido a que las partículas del agua residual están formadas en su mayoría por celulosa de papel, las mismas que tienden a pegarse entre sí formando flósculos de diámetro relativamente alto para que puedan precipitar con mayor facilidad. Sin embargo, esta concentración no es suficiente para poder descargar este efluente y según lo mostrado en la Tabla 1, referido al parámetro sólidos suspendidos, por lo que se requiere otro tratamiento, que es la filtración; la cual se la utilizará luego del reactor $\mathrm{RBC}$.

\subsubsection{Determinación del tiempo de sedimentación}

Para los tiempos de 70, 90 y 120 minutos de la experimentación, las concentraciones de sólidos sedimentables fueron de 207,7, 218,3 y 220,3 mL/L respectivamente. La diferencia entre los tiempos de 90 y 120 minutos es mínima de apenas de $2,0 \mathrm{~mL} / \mathrm{L}$, cinco veces menor con respecto al intervalo entre 70 y 90 minutos que fue de 10,6 $\mathrm{mL} / \mathrm{L}$. Por lo tanto, se concluye que el tiempo de sedimentación a escala de laboratorio es de 90 minutos.

\subsection{Evaluación del reactor biológico rotativo de contacto $(R B C)$ a escala de laboratorio}

3.3.1 Conteo y estabilización de los microorganismos en el $\mathrm{RBC}$

El promedio de la concentración de microrganismos presentes en la muestra del inóculo es de 20,4 x $10^{6} \mathrm{UFC} / \mathrm{mL}$, un valor adecuado que asegura la existencia de suficientes microorganismos para comenzar la estabilización microbiana en el RBC.

La variación de los parámetros de DBO y DQO en función del tiempo para el proceso de estabilización de los microorganismos en el reactor se observa en la Figura 3.

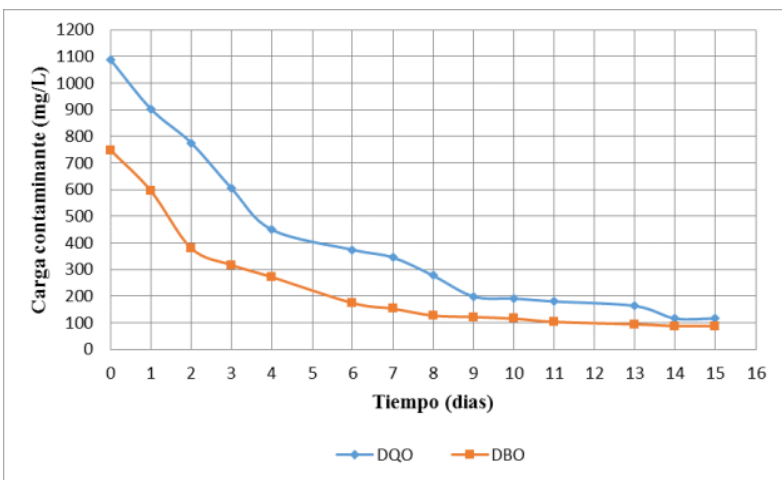

Figura 3. Variación de la $D B O$ y $D Q O$ en función del tiempo de estabilización de los microorganismos en el RBC

El tiempo de estabilización de los microorganismos fue de 14 días, un valor que se encuentra dentro del rango de bibliografía establecido por Castillo et al. (2012), el cual indica que se debe tomar entre 7 a 30 días (p. 85). El tiempo en tratamientos que interviene la formación de biopelícula es relativamente alto con respecto a otros tratamientos biológicos, debido a que la formación de la película de microorganismos entre el agua residual y la superficie de los discos requiere de un tiempo significativo.

\subsubsection{Determinación del flujo inicial de agua residual al RBC}

Se determinaron los caudales de las diferentes cargas orgánicas con valores de 35, 45, 55 y 60 g DQO/m²*día, los caudales fueron: $10,13,16$ y $18 \mathrm{~mL} / \mathrm{min}$ respectivamente. Los resultados de la concentración de la DQO al final del tratamiento facultativo se presentan en la Tabla 3.

Tabla 3. Resultados de los ensayos realizados a diferentes caudales de entrada al RBC para una DQO inicial de $1082 \mathrm{mg} / \mathrm{L}$

\begin{tabular}{cccc}
\hline \multirow{2}{*}{ Ensayo N. } & $\begin{array}{c}\text { Caudal de entrada } \\
\text { al RBC (mL/min) }\end{array}$ & $\begin{array}{c}\text { DQO del } \\
\text { afluente (mg/L) }\end{array}$ & $\begin{array}{c}\text { Remoción de } \\
\text { DQO (\%) }\end{array}$ \\
\hline 1 & 10 & 122,67 & 88,66 \\
2 & 13 & 187,77 & 82,65 \\
3 & 16 & 245,98 & 77,27 \\
4 & 18 & 383,67 & 64,54 \\
\hline
\end{tabular}

El caudal de $10 \mathrm{~mL} / \mathrm{min}$ muestra una mejor eficiencia de remoción de la DQO con un 88,66\%. Lo que demuestra una vez más que los microorganismos lograron estabilizarse de una forma adecuada con el agua residual de la fábrica productora de papel en el RBC.

\subsection{Evaluación de un filtro de arena y grava a escala de laboratorio}

3.4.1 Caracterización granulométrica de las arenas para el lecho filtrante

Se determinaron los coeficientes de uniformidad y de curvatura para cada muestra de arena de sílice: precipitada y fume. El coeficiente de uniformidad $(\mathrm{Cu})$ debe estar en un rango entre 0 y 2,5 para que el lecho filtrante no sufra grandes pérdidas de carga en el proceso de filtración con lo que se incrementa la eficiencia (Coulson y Richardson, 2003).

El valor de $\mathrm{Cu}$ para las arenas de sílice precipitada fue de 2,30, mientras que para las arenas de sílice fume fue de 3,10. Por lo 
tanto, la muestra de arena de sílice precipitada reportó un mejor coeficiente de uniformidad, lo que significa que tiene una mejor distribución granular con respecto a la muestra de arena fume; por lo tanto, se decidió que la arena de sílice precipitada se va usar como lecho filtrante para el filtro.

3.4.2 Determinación de la altura efectiva del filtro de arena y grava

La concentración de sólidos suspendidos del agua residual a la salida del filtro, para caudales de $20 \mathrm{~mL} / \mathrm{min}$ y $25 \mathrm{~mL} / \mathrm{min}$, en función de las alturas del lecho de la arena de sílice precipitada se observa en la Figura 4.

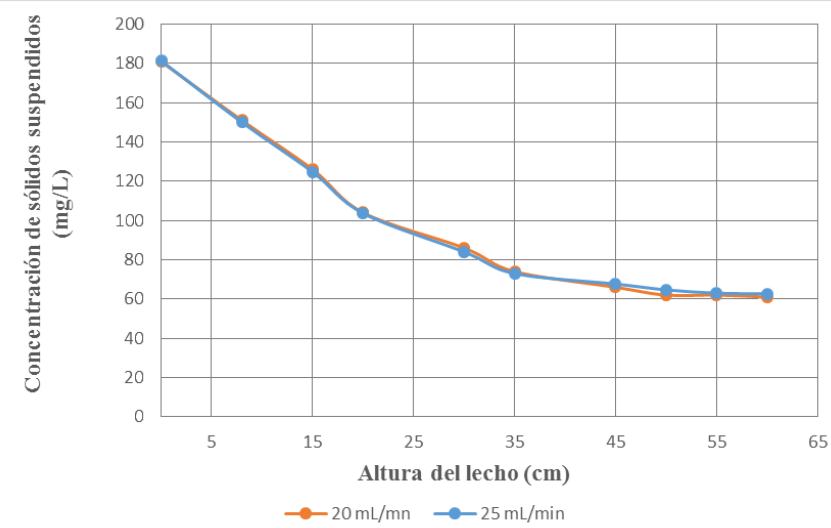

Figura 4. Concentración de sólidos suspendidos para diferentes alturas de lecho con caudales de entrada de agua residual de 20 y 25 mL/min

La remoción de sólidos suspendidos en función de la altura de lecho de arena de sílice precipitada, para los caudales de 20 y $25 \mathrm{~mL} / \mathrm{min}$, prácticamente tiene la misma tendencia. Dichas curvas se superponen debido a que la altura efectiva solo depende de la concentración inicial de sólidos y para ambos caudales es de $181 \mathrm{mg} / \mathrm{L}$ (Coulson y Richardson, 2003).

La altura efectiva es una variable de diseño importante para no sobredimensionar a un filtro. La prueba a escala de laboratorio, reportó una altura efectiva de $55 \mathrm{~cm}$ para los dos caudales, a partir de esta altura la concentración de sólidos se mantendrá constante con una concentración promedio de $62,5 \mathrm{mg} / \mathrm{L}$

3.4.3 Determinación del tiempo de saturación del filtro de arena y grava

Se construyó un filtro a escala de laboratorio con un diámetro de $0,15 \mathrm{~m}$, una altura de arena de sílice precipitada de $55 \mathrm{~cm}$ y grava como soporte del filtro. Otra variable de diseño importante es el tiempo de saturación del filtro para prevenir posibles pérdidas de carga y un mal funcionamiento del mismo (Romero, 2016). La tendencia de saturación del filtro por sólidos suspendidos en función del tiempo, para caudales de 20 y $25 \mathrm{~mL} / \mathrm{min}$, se observa en la Figura 5.

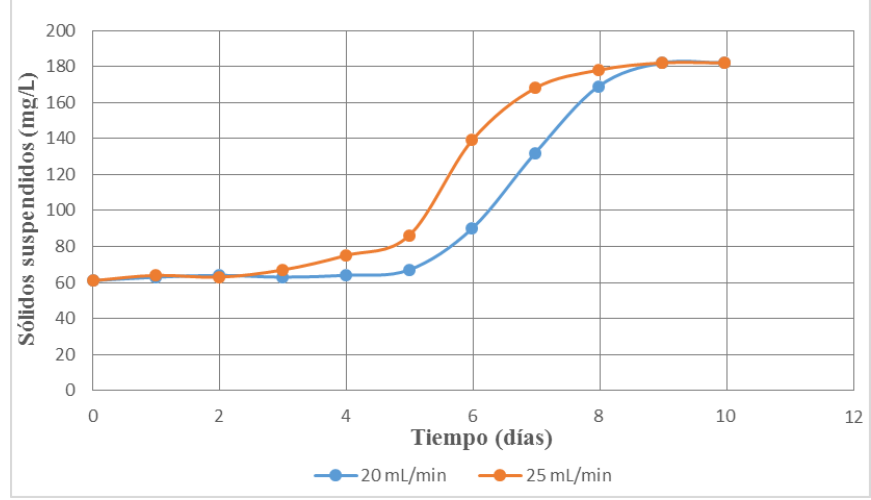

Figura 5. Tiempo de saturación del filtro de arena y grava a partir de caudales de 20 y $25 \mathrm{~mL} / \mathrm{min}$

Los resultados de la caracterización de los parámetros físicoquímicos del agua residual al inicio y final del sistema de tratamiento a escala de laboratorio, junto con 10 \% de remoción y la comparación de cumplimiento de norma se muestran en la Tabla 5.

Tabla 5. Caracterización inicial y final de los parámetros del agua residual luego del sistema de tratamiento a escala de laboratorio.

\begin{tabular}{ccrrrc}
\hline Parámetro & Unidad & $\begin{array}{c}\text { Valor } \\
\text { inicial }\end{array}$ & $\begin{array}{c}\text { Valor } \\
\text { final }\end{array}$ & $\begin{array}{c}\text { Remoción } \\
(\boldsymbol{\%})\end{array}$ & $\begin{array}{c}\text { Cumpli- } \\
\text { miento }\end{array}$ \\
\hline $\mathrm{DBO}$ & $\mathrm{mg} / \mathrm{L}$ & 980,2 & 88,2 & 91,01 & $\mathrm{SI}$ \\
$\mathrm{DQO}$ & $\mathrm{mg} / \mathrm{L}$ & 1707,0 & 116,2 & 93,14 & $\mathrm{SI}$ \\
$\begin{array}{c}\text { Sólidos totales } \\
\text { Sólidos } \\
\text { sedimentables }\end{array}$ & $\mathrm{mg} / \mathrm{L}$ & 6350,0 & 223,0 & 96,48 & $\mathrm{SI}$ \\
$\begin{array}{c}\text { Sólidos } \\
\text { suspendidos }\end{array}$ & $\mathrm{mg} / \mathrm{L}$ & 221,0 & 0,7 & 99,68 & $\mathrm{SI}$ \\
\hline
\end{tabular}

El agua residual proveniente de una industria de papel sometida a un sistema de descontaminación con base en los tratamientos de sedimentación, RBC y filtro granular, mostró una disminución en la concentración de los parámetros del agua residual con porcentajes superiores al $90 \%$ aproximadamente para: DBO, DQO, sólidos totales, sólidos sedimentables y sólidos suspendidos.

En la Tabla 6 se muestran los parámetros óptimos obtenidos a nivel de laboratorio para el tratamiento de aguas residuales de una fábrica de papel tisú.

Tabla 6. Parámetros óptimos a nivel de laboratorio para del tratamiento.

\begin{tabular}{lcc}
\hline \multicolumn{1}{c}{ Parámetro } & Valor & Unidad \\
\hline Tiempo de sedimentación & 90 & minutos \\
Concentración de microorganismos en el inóculo & $20,4 \times 10^{6}$ & $\mathrm{UFC} / \mathrm{mL}$ \\
Volumen inicial de agua residual en el RBC & 2,7 & $\mathrm{~L}$ \\
Volumen inicial de inóculo en el RBC & 0,3 & $\mathrm{~L}$ \\
Dosificación de agua residual para estabilizar RBC & 0,7 & $\mathrm{~mL} / \mathrm{min}$ \\
Tiempo de estabilización de m/o en RBC & 14 & días \\
Caudal de entrada de agua residual al RBC & 10 & $\mathrm{~mL} / \mathrm{min}$ \\
Altura del filtro de arena y grava & 55 & $\mathrm{~cm}$ \\
Diámetro del filtro de arena y grava & 15 & $\mathrm{~cm}$ \\
\hline
\end{tabular}

\subsection{Diseño de un sistema de tratamiento de aguas residuales}

Las operaciones unitarias del diagrama PFD se encuentran codificadas con letras y números para poder identificarlos (Páez, 2013). El diagrama de flujo PFD del sistema de tratamiento de aguas residuales se observa en la Figura 6. El control automático se implementó para tener un 
comportamiento predefinido y mejorar el desenvolvimiento del sistema (Sánchez, 2006).

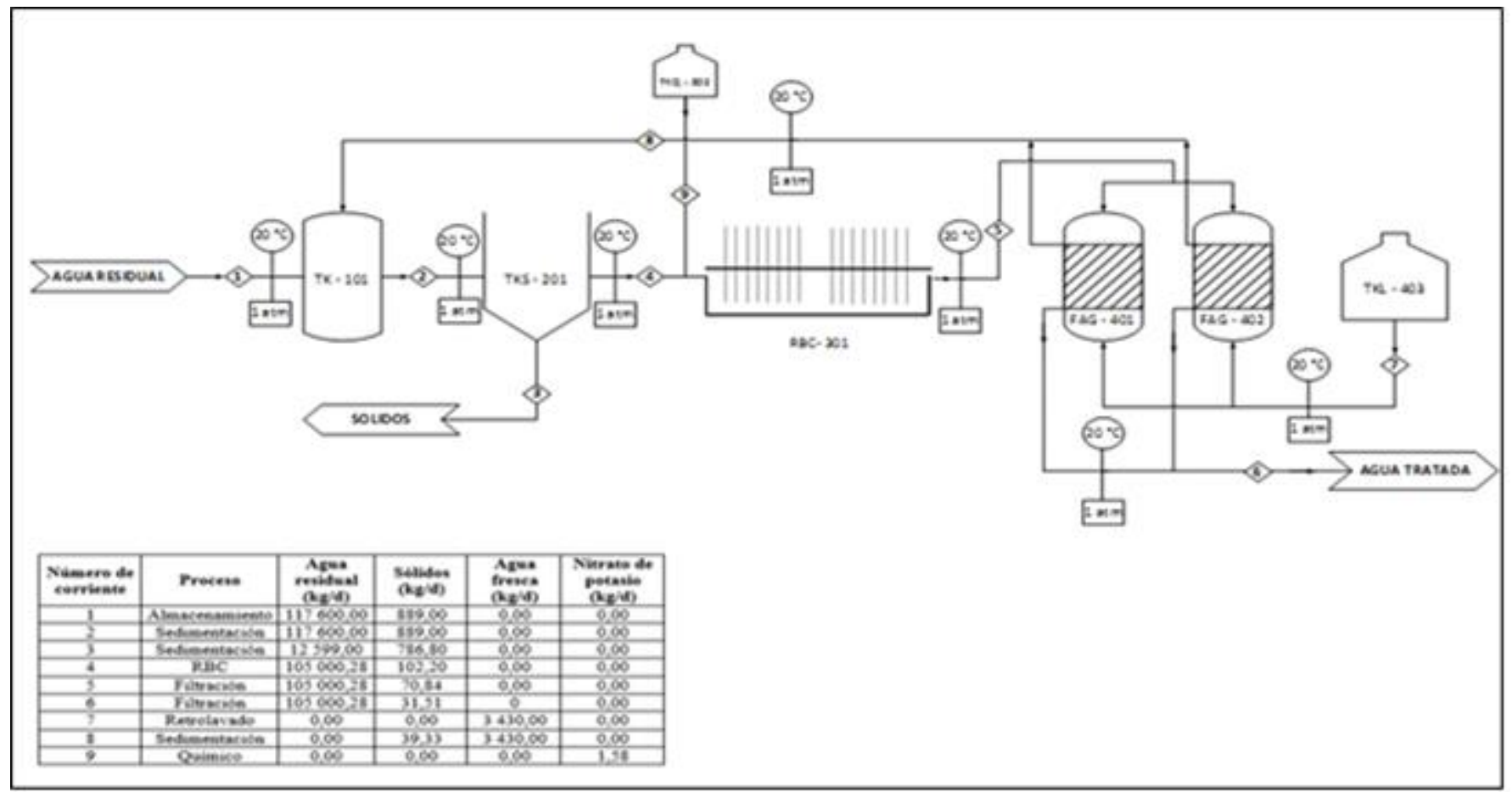

Figura 6. Diagrama de flujo del proceso para el tratamiento del agua residual de una industria de papel

\section{CONCLUSIONES}

Los parámetros iniciales físico-químicos del agua residual fuera de norma fueron: $980,2 \mathrm{mg} / \mathrm{L}$ para la DBO; $1707,0 \mathrm{mg} / \mathrm{L}$ para DQO; 6350,0 mg/L para sólidos totales; 4591,0 mg/L para sólidos suspendidos; $221 \mathrm{~mL} / \mathrm{L}$ para sólidos sedimentables. Estos valores superan el límite máximo permisible para descargas líquidas hacia un cuerpo de agua dulce, cuerpo de agua marina o alcantarillado público, destallado en el Registro Oficial $\mathrm{N}^{\circ} 387$ del 4 de Noviembre del 2015 de la República del Ecuador.

El conteo inicial de los microorganismos presentes en el inóculo procedente de una industria maderera fue de 20,41E+06 aproximadamente. Este valor fue óptimo para empezar el proceso de estabilización microbiana en el reactor biológico rotativo de contacto.

La estabilización del inóculo de microorganismos con el agua residual para una concentración inicial del $10 \% \mathrm{v} / \mathrm{v}$, tuvo un tiempo de 14 días dentro del rango establecido en bibliografía. Así pues, se demostró que la compatibilidad entre el inóculo y el agua residual en el reactor biológico rotativo fue adecuada.

En el reactor biológico rotativo de contacto a escala de laboratorio, el mejor flujo de agua residual a la entrada fue de $10 \mathrm{~mL} / \mathrm{min}$ con un porcentaje de reducción del 88,66 \% para la DQO.

La altura efectiva del lecho de arena a escala de laboratorio fue de $55 \mathrm{~cm}$ y presentó una remoción de sólidos suspendidos del $66,75 \%$.
El sistema de tratamiento con base en un reactor biológico rotativo de contacto, obtuvo una eficiencia de depuración del $91,01 \%$ para la DBO; $93,14 \%$ para la DQO; $96,48 \%$ para los sólidos totales; 99,68\% para los sólidos sedimentables y 99,66 $\%$ para sólidos suspendidos, por lo que permitió el cumplimiento con las normas técnicas para el control de descargas líquidas.

\section{REFERENCIAS}

APHA, A. P. (2012). Standard methods for the examination of water (22va Ed). New York, EEUU.

Behling, E., Colina, G., Díaz, A., Marín, J., Rincón, N., y Fernández, N. (2008). Tratamiento Biológico de Aguas Residuales Industriales: Efluente Camaronero en Reactores RBC. Boletín del Centro de Investigaciones Biológicas, 42(2), 243-255

Casey, P.J. (2000). Pulpa y papel: Química y tecnología química. (4ta. Ed.). Madrid, España: LIMUSA S. A.

Castillo, E., Bolio, A., Méndez, R, Osorio, J., y Pat, R. (2012). Remoción de materia orgánica en aguas residuales de rastro por el proceso de Contactor Biológico Rotativo. Ingeniería, Revista Académica de la FI-UADY, 16(2), 83-91

Coulson, J. M., y Richardson, J. F. (2003). Ingeniería química. Tomo II: Operaciones básicas. (2da. Ed.). Barcelona, España: Reverté.

Correa, R., Tapia, L. (2015). Registro Oficial N 387 del 4 de Noviembre del 2015 de la República del Ecuador. Recuperado de: http://extwprlegs1.fao.org/docs/pdf/ecu155142.pdf. (Enero, 2018)

Davis, M. (2010). Wastewater engineering: Sedimentation. (1era. Ed.). New York, Estados Unidos: McGraw-Hill.

Deloya, A. (2001). Biodiscos, una alternativa de tratamiento biológico para aguas residuales cuando no se disponen de grandes extensiones de terreno. Tecnología en marcha, 13(3), 57-59

GAB System (2005). Cámara Thoma y Neubauer improved para el recuento de levaduras (tiraje). Obtenido de: http://shop.gabsystem.com/data/descargas/Camara\%20Thoma\%20Neub auer_SP.pdf. (Abril, 2017)

Metcalf, L., y Eddy, H. (2003). Tratamiento y depuración de las aguas residuales. (4ta ed.). New York, Estados Unidos: McGraw-Hill. 
Páez, M. (2013). Manual para el estudiante de diseño de plantas industriales (1era. ed.). Quito, Ecuador: EPN.

Romero, J. (2016). Tratamiento de aguas residuales: Teoría y principios de diseño. (3era. Ed). Bogotá, Colombia: Escuela Colombiana de Ingeniería.

Sánchez, J. (2006). Instrumentación y control avanzado de procesos (1era. ed.). Madrid, España: Díaz de Santos.

\section{BIOGRAFÍAS}

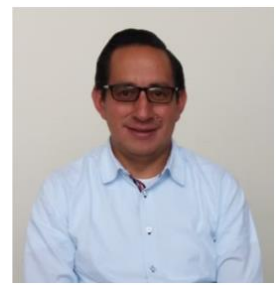

Marcelo F. Cabrera J. Nacido el 05 de marzo de 1986 en Quito-Ecuador, fue el mejor graduado de la Unidad Educativa "Jean Jacques Rousseau", promoción 2003-2004. Continuó sus estudios de pregrado en la Escuela Politécnica Nacional, obteniendo el título de Ingeniero Químico Aprobado Cum Laude. Finalmente obtuvo su título de posgrado en la Universidad Internacional SEK como Magíster en Gestión Ambiental. Ha trabajado en Incinerox, Chemeng y Escuela Politécnica Nacional donde hasta la actualidad labora como docente en la Facultad de Ingeniería Química y Agroindustria.

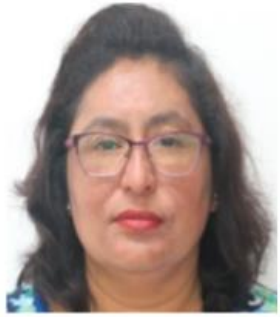

Lucía Montenegro Aguas. Ingeniera Química, Magister en Ingeniería Ambiental ha sido docente por 24 años en la EPN de las cátedras de Transferencia de Calor, Control de la Contaminación del Aire entre otras. Cuenta con la Certificación de Evaluador de la Norma ISO 17025 para laboratorios. En referencia a las publicaciones las ha realizado en la "Revista Politécnica" y en la "1st International Conference on Water and Sustainability" con temas relacionados al Tratamiento de aguas y Remediación de Suelos. Ha dictado más de 20 seminarios y conferencias de Monitoreo Ambiental; y dirigido más de 40 proyectos de titulación en temas de Transferencia de Calor, Refinación del Petróleo y Contaminación Ambiental.

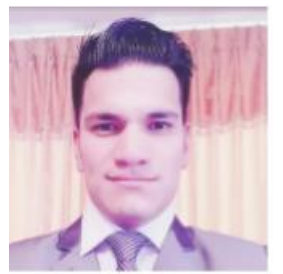

Edgar Omar Merizalde Acuña. Nacido en Quito el 19 de noviembre de 1988. Actualmente llevo dos años de casado y tengo una hija de un año tres meses. Me gradué de ingeniero Químico de la Escuela Politécnica Nacional en diciembre del 2015 y de Master en dirección de operaciones y calidad de la Universidad Internacional de la Rioja en marzo del 2019. Mi profesión se ha enfocado tanto en la investigación y desarrollo como en la mejora de procesos. Elaboré del 2015-2017 como analista de procesos en Familia Sancela, del 2017-2018 como analista de investigación y desarrollo en laboratorios Rene Chardon y desde el 2017 hasta la actualidad como analista líder de Pharmaceutical Technology Development en Grunenthal Ecuador. 